\title{
iPS 细胞的遗传安全性
}

陈倩，史庆华

中国科学技术大学生命科学学院, 合肥微尺度物质科学国家实验室, 合肥 230027

摘要: 自 2006 年 Takahashi 和 Yamanaka 首次成功地从小鼠成纤维细胞诱导得到诱导多能性干细胞(Induced pluripotent stem cells, iPS 细胞) 以来, iPS 细胞由于其潜在的广阔应用前景而迅速成为干细胞研究领域的新热点; 与此同时, iPS 细胞的遗传安全性也越来越多地受到人们的关注。文章将对 iPS 细胞遗传安全性的研究进展进行 综述, 分析造成 iPS 细胞遗传不稳定的可能原因, 希望可以促进对iPS 细胞诱导条件的优化, 获得遗传上较为安 全的 iPS 细胞。

关键词：iPS 细胞；遗传安全性；遗传不稳定；遗传异常；表观遗传异常

\section{The genetic safety of induced pluripotent stem (iPS) cells}

\section{CHEN Qian, SHI Qing-Hua}

Hefei National Laboratory for Physical Sciences at Microscale, School of Life Sciences, University of Science and Technology of China, Hefei 230027, China

\begin{abstract}
Since Takahashi and Yamanaka first generated induced pluripotent stem (iPS) cells from mouse fibroblasts successfully in 2006, iPS cells have rapidly become a new hotspot in the field of stem cells research because of their broad potential application prospects. Meanwhile, more and more attentions are paid to the genetic safety of iPS cells. This article summarizes recent findings on genetic safety of iPS cells and reviews the possible causes leading to genetic instability of iPS cells. Hopefully, this review is helpful to improve the induction of iPS cells and obtain genetically safe iPS cells.
\end{abstract}

Keywords: iPS cells; genetic safety; genetic instability; genetic abnormality; epigenetic abnormality

2006 年, 日本京都大学的 Takahashi 和 Yamanaka ${ }^{[1]}$ 首次运用逆转录病毒感染技术, 把 Oct3/4、Sox2、Klf4 和cMyc 4 种转录因子导入胚胎 或成年小鼠的成纤维细胞中, 成功地将体细胞诱导 成与胚胎干细胞极为相似的多能性干细胞, 并命名
为诱导多能性干细胞(Induced pluripotent stem cells, iPS细胞)。相对于胚胎干细胞(Embryonic stem cells, ES细胞), iPS细胞的产生不需要卵母细胞或早期胚 胎细胞，因此不涉及胚胎毁损等伦理问题; 同时, 利用自体来源的iPS细胞进行移植治疗也可避免异

\footnotetext{
收稿日期: 2011-11-16; 修回日期: 2012-01-12

基金项目: 中国科学院“百人计划”项目(编号：KJ207004)和国家自然科学基金项目(编号：30711120571)资助

作者简介: 陈倩, 硕士, 专业方向：iPS 细胞的细胞分子遗传学。E-mail: cendy@mail.ustc.edu.cn

通讯作者: 史庆华, 博士, 教授, 研究方向：非整倍体细胞的发生、命运及其细胞分子生物学机制和卵子发生和卵泡形成的分子调控。E-mail： qshi@ustc.edu.cn

致谢: 感谢黄韵、易启毅、赵小玉和江龙(中国科学技术大学生命科学学院分子细胞遗传学实验室)提供的宝贵修改意见。

网络出版时间: 2012-2-13 16:44:03

URL: http://www.cnki.net/kcms/detail/11.1913.R.20120213.1644.005.html
} 
体间的免疫排斥。因此, iPS细胞的出现实现了伦理 和技术上的双重突破，成为继克隆绵羊“多莉 (Dolly)”和人ES细胞建系之后干细胞研究领域新的 里程碑。近年来, 有关iPS细胞的研究呈爆炸性增长, 并取得了一系列突破性的进展。但与此同时, iPS细 胞的遗传安全性问题也越来越多地受到人们的关 注。本文将对iPS细胞遗传安全性的研究进行总结, 并分析导致iPS细胞遗传不稳定的可能原因, 以期为 获得安全性较高的iPS细胞提供依据。

\section{1 遗传安全是 iPS 细胞应用的前提}

遗传上安全的iPS细胞应具有正常的并可稳定 传递给子代的遗传学和表观遗传学组成, 从而保证 细胞能行使正常的功能。遗传学异常是指基因突变 或染色体变异(包括染色体数目异常和结构畸变)。表 观遗传学异常则是遗传物质本身未发生变异, 但遗 传物质的表达出现异常, 通常由DNA或组蛋白的异 常修饰引起。无论是遗传学异常还是表观遗传学异 常, 都有可能引起细胞和机体的结构或功能的改变, 甚至导致疾病的发生。例如, 高达 $92 \%$ 的人类实体 瘤都是非整倍体 (即发生染色体数目异常) $)^{[2]}$, 并且 肿瘤中非整倍体细胞的比例与肿瘤的恶化进程、转 移风险、治疗效果以及复发率等密切相关 ${ }^{[3]}$ 。而许 多造血系统的肿瘤细胞都具有特异的染色体结构异 常, 如 $\mathrm{Ph}$ 染色体是慢性粒细胞白血病的标志性染色 体。在表观遗传学水平, DNA甲基化状态的改变则可 能影响癌症发生相关基因的表达。DNA的低甲基化 可能会激活癌基因, 而高甲基化则可能引起抑癌基 因沉默。因此, iPS细胞研究和应用的前提不仅是具 有无限增殖的能力和多向分化的潜能, 而且在诱 导、增殖和分化过程中都必须保持正常且稳定的遗 传学和表观遗传学组成。

\section{2 iPS 细胞的遗传组成}

自从第一次成功诱导 iPS 细胞, 人们就开始关 注 iPS 细胞的遗传组成。迄今为止, 包括我们实验室 在内的众多实验室采用各种不同的检测方法, 对多 种 iPS 细胞的遗传安全性进行了检测。例如, 用 G带核型分析、光谱核型分析 (Spectral karyotyping, SKY)和苂光原位杂交(Fluorescence in situ hybridiza- tion, FISH)来检测染色体组成, 用 Array-based 比较 基因组杂交 (Comparative genomic hybridization, $\mathrm{CGH}$ )检测 DNA 序列的拷贝数变化(Copy number variations, CNVs), 以单核苷酸多态性芯片(Singlenucleotide polymorphism-based microarrays, SNP 芯 片)来同时检测 CNVs 和基因的杂合丢失。此外, 外 显子测序和 DNA 甲基化测序等技术，也用于 iPS 细 胞的遗传和表观遗传学分析。但是, 研究结果却不 尽相同(表 1)。

值得注意的是, 所有认为 iPS 细胞具有正常稳 定遗传组成的研究, 均采用了传统细胞遗传学分析 技术(Giemsa、Hoechst/DAPI 染色或 G-带核型分析), 而所有利用现代分子遗传学分析技术进行的研究, 都在 iPS 细胞中发现了不同种类和不同程度的遗传 或表观遗传异常。

在Minina等 ${ }^{[171}$ 第一个针对iPS细胞的染色体稳 定性进行细致地研究之前, 全世界的iPS细胞研究者 都将主要的精力集中在开发新的iPS细胞诱导方法 及其应用上, 而对iPS细胞遗传安全性的研究只是附 带观察所获得的iPS细胞染色体是否正常 ${ }^{[1,13 ~ 15,18]}$ 。而 即使在iPS细胞中观察到一些遗传异常也没有引起 人们足够的重视, 并普遍认为iPS细胞在遗传上是安 全的。

Minina等 ${ }^{[17]}$ 首次针对iPS细胞的染色体稳定性 进行了研究, 发现所研究的两个细胞系均存在与 Takahashi和Yamanaka的报道相似的X染色体单体, 并在其中 1 个细胞系中发现了染色体的重排(Rearrangement)和断片(Fragments)。随后, Mayshar等 ${ }^{[16]}$ 对多 种iPS细胞的染色体组成进行了系统研究, 发现在人 源的 66 个iPS(Human iPS, hiPS)细胞系中, 大多数都 涉及部分或整条染色体的畸变。之后, iPS细胞遗传 安全性迅速成为iPS细胞研究领域的热点之一, 并获 得了一系列令人忧心的结果, 现总结如下:

第一, iPS细胞比ES细胞或产生iPS细胞的体细 胞具有更多的遗传学和表观遗传学异常, 包括 : (1) 染色体异常。Pasi等 ${ }^{[23]}$ 发现在iPS细胞中染色体异常 显著增加。我们对 9 个小鼠iPS(Mouse iPS, miPS)细 胞系进行的研究也表明, 其中 7 个细胞系都出现了 程度不同的染色体异常 ${ }^{[25]}$ 。但用于诱导产生这些 miPS细胞系的体细胞核型均正常。(2)基因拷贝数变 
表 1 iPS 细胞的遗传安全性研究总结

\begin{tabular}{|c|c|c|c|c|}
\hline 细胞 & 诱导方法 & 检测方法 & 检测结果 & 文献 \\
\hline hiPSCs & $\begin{array}{l}\text { 含 Oct4、Sox2、Nanog 和 } \operatorname{Lin} 28 \text { 的 } \\
\text { 慢病毒感染 }\end{array}$ & G-带核型 & 核型正常 & {$[4]$} \\
\hline miPSCs & $\begin{array}{l}\text { 含 Oct4、Sox2、Klf4 和 } n M y c \text { 的慢 } \\
\text { 病毒感染 }\end{array}$ & 中期相 & 核型正常 & [5] \\
\hline hiPSCs & $\begin{array}{l}\text { 含 Oct4、Sox2、Klf4 和 Myc 的逆转 } \\
\text { 录病毒感染 }\end{array}$ & G-带核型 & 核型正常 & {$[6]$} \\
\hline miPSCs & $\begin{array}{l}\text { 含 Oct4、Sox2、Klf4 和 } M y c \text { 的逆转 } \\
\text { 录病毒感染 }\end{array}$ & DAPI 中期相染色 & 核型正常 & [7] \\
\hline hiPSCs & $\begin{array}{l}\text { 含 Oct4、Sox2、Nanog、Lin28、cMyc } \\
\text { 和 Klf4 的质粒(oriP/EBNA1 载体) }\end{array}$ & G-带核型 & 核型正常 & [8] \\
\hline $\begin{array}{l}\text { miPSCs } \\
\text { hiPSCs }\end{array}$ & $\begin{array}{l}\text { 用 } 2 \mathrm{~A} \text { 自我剪接肽及同 } 1 \text { 个启动子 } \\
\text { 表达 Oct4、Sox2、Klf4 和 } c M y c \text { 的 } \\
\text { 慢病毒载体 }\end{array}$ & G-带核型 & 核型正常 & [9] \\
\hline $\begin{array}{l}\text { riPSCs } \\
\text { hiPSCs }\end{array}$ & $\begin{array}{l}\text { riPSCs:Oct4、Sox2 和 Klf4 逆转录 } \\
\text { 病毒; hiPSCs:Oct4、Sox2、Nanog } \\
\text { 和 Lin28 慢病毒; 添加 PD0325901、 } \\
\text { A-83-01 和 CHIR99021 }\end{array}$ & G-带核型 & 核型正常 & [10] \\
\hline hiPSCs & 直接添加重编程蛋白 & G-带核型 & 与起始体细胞核型一样 & [11] \\
\hline miPSCs & 单次导入胚胎干细胞衍生蛋白 & G-带核型 & 核型正常 & [12] \\
\hline miPSCs & $\begin{array}{l}\text { Oct3/4、Sox2、cMyc T58A 突变体/ } \\
\text { 野生型和 Klf4; 逆转录病毒感染 }\end{array}$ & Q-带分析 & $\begin{array}{l}5 \text { 个iPS-TTFgfp 4(cMyc T58A 突变)克隆中, } 3 \text { 个核 } \\
\text { 型异常, 分别为 } 39 X \mathrm{XO} \text { 、 } 40 \mathrm{XO}+10 、 40 \mathrm{Xi}(\mathrm{X}) ; 3 \text { 个 } \\
\text { iPS-TTFgfpWT(cMyc 野生型)克隆均核型正常。 }\end{array}$ & [1] \\
\hline miPSCs & $\begin{array}{l}\text { 含 Oct4、Sox2、Klf4 和 Myc 的逆转 } \\
\text { 录病毒感染 }\end{array}$ & G-带核型 & 20\%细胞系核型异常 & [13] \\
\hline hiPSCs & $\begin{array}{l}4 \text { 因子:Oct4、Sox2、Klf4 和 } c M y c \\
\text { 或 Oct4、Sox2、Nanog 和 Lin28; } \\
\text { 慢病毒; 添加 SV40 大 T 抗原提高 } \\
\text { 诱导效率 }\end{array}$ & G-带核型 & 10 代后, 90\%的细胞核型异常 & [14] \\
\hline miPSCs & $\begin{array}{l}\text { Oct4、Sox2、Klf4 和 } c M y c \text { 腺病毒 } \\
\text { 感染 }\end{array}$ & DNA 含量分析 & $23 \%$ 的 adeno-iPS 细胞系是四倍体 & [15] \\
\hline hiPSCs & $\begin{array}{l}\text { 多种诱导方法(来自多个实验室的 } \\
66 \text { 个细胞系) }\end{array}$ & $\begin{array}{l}\mathrm{G} \text {-带核型, CGH, SNP, 全基因表 } \\
\text { 达谱分析(Expander 和 EASE 软件) }\end{array}$ & $\begin{array}{l}\text { 大量细胞系中出现涉及整条染色体和染色体片 } \\
\text { 段的畸变和高频率的 } 12 \text { 号染色体重复。 }\end{array}$ & [16] \\
\hline miPSCs & $\begin{array}{l}\text { Oct4、Sox2、Klf4 和 } c M y c \text { 慢病毒 } \\
\text { 感染 }\end{array}$ & G-带核型 & $\begin{array}{l}2 \text { 个 } i P S \text { 细胞系均为 } X \text { 染色体单体, 其中 } 1 \text { 个 iPS } \\
\text { 细胞系检测到染色体重排和染色体断片。 }\end{array}$ & [17] \\
\hline miPSCs & $\begin{array}{l}\text { Oct4、Sox2、Klf4 和/或 } c M y c \text {, 可剪 } \\
\text { 切的多顺反子慢病毒载体, 然后用 } \\
\text { 表达 Cre 重组酶的腺病毒载体 } \\
\text { (Adeno-Cre)将重编程转基因切除 }\end{array}$ & SKY & $\begin{array}{l}\text { iPS 细胞克隆中未发现染色体易位, 但 } 25 \%(2 / 8) \\
\text { OSKM 克隆和 } 11 \%(1 / 9) \text { OSK 克隆出现 } 8 \text { 号染色 } \\
\text { 体三体。 }\end{array}$ & [18] \\
\hline hiPSCs & $\begin{array}{l}4 \text { 因子逆转录病毒; } 4 \text { 因子慢病毒; } 3 \\
\text { 因子逆转录病毒; 游离载体和 } \\
\text { mRNA(22 个细胞系) }\end{array}$ & $\begin{array}{l}\text { G-带核型; 用 padlock probes、 } \\
\text { in-solution DNA or RNA baits 对 } \\
\text { 外显子测序 }\end{array}$ & $\begin{array}{l}\text { 所有细胞系核型均正常; 但每个 hiPS 细胞系在 } \\
\text { 检测的区域平均发生 } 5 \text { 个点突变; hiPS 细胞除发 } \\
\text { 生表观遗传学改变, 还出现了遗传改变。 }\end{array}$ & [19] \\
\hline hiPSCs & $\begin{array}{l}\text { Oct4、Sox2、Klf4、Nanog 和 Lin28 } \\
\text { 通过逆转录病毒或 piggyBac(22 个 } \\
\text { 细胞系) }\end{array}$ & $\begin{array}{l}\text { Affymetrix SNP array } 6.0, \mathrm{CNVs} \\
\text { 分析 }\end{array}$ & $\begin{array}{l}\text { 早代 hiPS 细胞 CNVs 的数量比晚代 hiPS 细胞以 } \\
\text { 及成纤维细胞和 hES 细胞显著增多。 }\end{array}$ & [20] \\
\hline hiPSCs & $\begin{array}{l}\text { 多种诱导方法(186 个多能性干细 } \\
\text { 胞系) }\end{array}$ & 高分辨率的 SNP & $\begin{array}{l}\text { 在多能性相关基因和假基因中有频繁的 CNVs; } \\
\mathrm{CNVs} \text { 的频率总体升高。 }\end{array}$ & [21] \\
\hline hiPSCs & 5 个 hiPSCs 细胞系 & $\begin{array}{l}\text { 单碱基分辨率的全基因组检测 } \\
\text { 技术 }\end{array}$ & $\begin{array}{l}\text { iPS 和 ES 细胞系之间存在 } 1000 \text { 多个不同的甲基 } \\
\text { 化区域, 主要分为 : CG 二核苷酸的异常甲基化 } \\
\text { 和在非 CG 区的异常甲基化。 }\end{array}$ & [22] \\
\hline
\end{tabular}


续表 1

\begin{tabular}{|c|c|c|c|c|}
\hline 细胞 & 诱导方法 & 检测方法 & 检测结果 & 文献 \\
\hline miPSCs & $\begin{array}{l}\text { Oct4、Sox2、Klf4 和 } c M y c(\mathrm{OSKC}) \text {; } \\
\text { Oct4、Sox2 和 Klf4(OSK) 慢病毒感染 }\end{array}$ & 核型分析和 CGH & $\begin{array}{l}\text { 所有 OSK 克隆(6)均为二倍体, } 50 \% \text { OSKC 克隆为 } \\
\text { 非整倍体。33\%(2/6)OSK 克隆在 } 8 \text { 号染色体上有 } \\
\text { 小片段获得; } 1 \text { 个二倍体 OSKC 克隆在 } 7 \text { 号染色体 } \\
\text { 上有扩增而在 } 8 \text { 号染色体上有缺失。 }\end{array}$ & [23] \\
\hline hiPSCs & Oct4、Sox2、Klf4 和 cMyc 逆转录病毒 & 染色体计数, SKY 和 FISH & 检查的所有 hiPS 细胞群体都含有非整倍体细胞。 & [24] \\
\hline miPSCs & $\begin{array}{l}\text { Oct4、Sox2、Klf4 和 } c M y c(\mathrm{OSKM}) \text {; } \\
\text { Oct4、Sox2 和 Klf4(OSK); Oct4、Sox2、 } \\
\text { Klf4 和 Nanog(OSKN) 逆转录病毒感染 }\end{array}$ & G-带核型分析, SKY 和 FISH & $\begin{array}{l}\text { 在检查的 } 9 \text { 个细胞系中, } 2 \text { 个核型正常, } 7 \text { 个含有比 } \\
\text { 例不同的异常核型细胞, 其中 } 6 \text { 个具有 } 14 \text { 号染色 } \\
\text { 体三体和/或罗伯逊易位; OSKM 细胞系中罗伯逊 } \\
\text { 易位的发生率显著高于 OSK 和 OSKN 细胞系。 }\end{array}$ & [25] \\
\hline
\end{tabular}

异。Hussein等 ${ }^{[20]}$ 在iPS细胞中发现了大量的、比亲 代成纤维细胞或 ES 细胞中高约两倍的 CNVs。 Laurent等 ${ }^{[21]}$ 发现在iPS细胞中, 其与多能性相关的 基因和假基因中频繁出现CNVs。(3)点突变。Gore 等 ${ }^{[19]}$ 发现无论iPS细胞的诱导方法是否会导致基因 整合, 但诱导产生的每个iPS细胞克隆中平均存在 5 个蛋白质编码序列的点突变。(4)表观遗传学变异。 Lister等 ${ }^{[22]}$ 发现iPS细胞和ES细胞有 1000 多个甲基 化区域上的差异(Differentially methylated regions, DMRs), 包括在 $C G$ 二核苷酸区的异常甲基化和在非 CG区的异常甲基化，而后者则是iPS细胞特有的表 观遗传学特征。他们进一步发现iPS细胞中的一些 DMRs能传递给由其分化形成的子细胞。因此iPS细 胞中存在着多个水平的遗传不安全性。

第二, 在iPS细胞产生的不同阶段都可发生遗传 学异常。如：(1)一些遗传损伤来源于诱导产生iPS

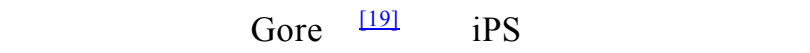
突变也存在于用来诱导产生iPS细胞的体细胞中。(2) 一些遗传异常出现在诱导过程中。Hussein等 ${ }^{[20]}$ 认为, 诱导过程本身可能会导致 CNVs的显著增加, 以致 诱导产生的iPS细胞具有大量的新生CNVs。Laurent 等 ${ }^{[21]}$ 的研究表明, iPS和ES细胞基因组异常的模式不 同，提示诱导过程也可能导致了iPS细胞的基因变 异。Pasi等 ${ }^{[23]}$ 进一步发现, 诱导因子 $M y c$ 的使用与 $\mathrm{iPS}$ 细胞的染色体异常有关, 因为单独利用 $M y c$ 将乳腺 祖细胞诱导为iPS细胞的过程中伴随着染色体异常 的高发。我们的研究也表明, $c M y c$ 参与诱导产生的 miPS细胞系相对于无 $c M y c$ 参与诱导产生的miPS细 胞系, 其染色体发生罗伯逊易位的频率显著增高 [25]。显然, 诱导过程本身可损害iPS细胞的遗传安全 性。(3)还有一些遗传异常与细胞对长 期培养的适应
有关。Hussein等 ${ }^{[20]}$ 发现iPS细胞在传代培养过程中 $\mathrm{CNVs}$ 出现动态变化, 且在传代培养后期 CNVs的发 生率降低, 推测大部分新生的CNVs对iPS细胞有害 而导致相应的iPS细胞被淘汰，引起传代培养后期 iPS细胞中CNVs的发生率下降。Laurent等 ${ }^{[21]}$ 认为细 胞培养时间的延长会导致iPS和ES细胞中基因变异 的积累。我们的研究也表明, 核型异常的miPS细胞 在长期培养过程中, 多种核型异常细胞的比例会逐 渐增多或减少 ${ }^{[25]}$ 。因此, 体外长期培养可导致细胞 遗传结构的改变, 不利于细胞成活和生长的遗传改 变被逐渐淘汰, 而有利于细胞存活和生长的遗传改 变被篮选保留下来。

第三，iPS细胞中出现的遗传损伤具有潜在的致 癌性。例如, 易发生基因扩增、缺失或点突变的区域 通常富含细胞周期调控基因和癌基因 ${ }^{[21]}$ 。Gore等 ${ }^{[19]}$ 发 现在iPS细胞克隆中, 有些突变发生于肿瘤发生相关 基因如 $A T M$, 提示iPS细胞中的遗传损伤可能与肿瘤 发生有关。我们的研究也表明, 具有特定核型的 miPS细胞相对于正常核型的细胞具有增殖优势，其 进入有丝分裂的能力显著增强 ${ }^{[25]}$ 。有研究表明, 多 能性干细胞增殖能力的增强常伴随分化潜能的减弱, 在体内易引发肿瘤。如, Okita、Nakagawa和Nagata 研究组均报道, 有 $c M y c$ 参与诱导的iPS细胞所产生的 嵌合体小鼠易发肿瘤, 死亡率较高 ${ }^{[26-28]}$; 而 Pasi等 ${ }^{[23]}$ 和我们 ${ }^{[25]}$ 的研究都发现, 有 $c M y c$ 参与诱导的iPS细 胞的染色体异常发生率较高。这些结果提示, iPS细 胞产生的嵌合体小鼠的肿瘤发生增加可能与iPS细 胞的遗传异常有关。因此, 在iPS细胞应用之前, 必 须确保其遗传完整性和安全性。

综上所述, iPS 细胞可在染色体、亚染色体和单 碱基水平上发生多种遗传变异, 这些变异可发生于 
iPS 细胞的诱导和随后的传代培养过程中, 并与细 胞的增殖有关。此外, 不同的检测方法对 iPS 细胞遗 传稳定性的检测结果也不尽相同, 现代分子遗传学 分析技术较传统细胞遗传学分析技术发现了更多的 iPS 细胞遗传异常, 这可能与所用检测方法的分辨 率不同有关。传统细胞遗传学分析, 只能从染色体 或染色体区段的水平对遗传物质的完整与否进行评 价，而 CGH 和 SNP 芯片杂交分析以及外显子测序则 是从 DNA 序列和单碱基水平, 对遗传物质的组成进 行检测。但总的来说, iPS 细胞具有遗传和表观遗传 异常是布庸质疑的。

\section{3 导致 iPS 细胞遗传异常的可能因素}

导致 iPS 细胞发生遗传异常的因素很多，如产 生 iPS 细胞的体细胞、诱导因子本身的性质、诱导 因子导入细胞的方式、诱导因子及其载体在染色体 上的整合位点以及诱导过程和诱导完成后的细胞培 养等。

\section{1 iPS 细胞的诱导}

\subsection{1 起始体细胞群体}

自iPS细胞从小鼠的胚胎成纤维细胞第一次成 功诱导以来, 成纤维细胞一直是被优先地用于产生 iPS细胞的起始细胞, 这主要是由于它们的分离和培 养都比较容易。但也有很多其他类型的体细胞被成 功地诱导成为iPS细胞的报道，如胃上皮细胞、肠上 皮细胞、肝细胞、胰脏 $\beta$ 细胞、淋巴细胞、肌细胞和 肾上腺细胞等。不同类型的体细胞对于诱导因子的 导入、诱导的动力学和效率都有很大影响 ${ }^{[29]}$ 。如诱 导产生iPS细胞所需的病毒滴度在不同类型的体细 胞间差异很大，小鼠成纤维细胞所需的腺病毒滴度 比胎鼠肝细胞要高 $100 \sim 200$ 倍 ${ }^{[15]}$ 。胃上皮细胞和肝
细胞较成纤维细胞可被更快地诱导为iPS细 胞 ${ }^{[29 .}$ 30]。需要强调的是, 体细胞的遗传安全性将直接影响 由其诱导产生的iPS细胞的遗传安全性, 而不同来源 的体细胞，由于其供体年龄不同等，细胞内染色体 异常的发生率和DNA的损伤程度也不尽一致 ${ }^{[31,32]}$ 。因 此, 选择起始细胞群体需要综合考虑各方面的因素, 如细胞类型、供体年龄、所需病毒滴度和诱导效率 等。

\subsection{2 诱导因子的载体}

如表 2 所示, 无论是利用逆转录病毒、慢病毒、 腺病毒、转座子, 还是瞬时转染, 都是以核酸为基础 的导入方式，都涉及载体在细胞基因组中的整合。 使用逆转录病毒或慢病毒为载体诱导产生的iPS细 胞, 其基因组上很可能包含多个基因整合位点, 容 易引发插入突变。腺病毒能将外源基因以非插入的 方式导入细胞中，而且外源基因在不整合的情况下 也能表达, 这可显著降低由于插入导致基因突变的 风险，然而腺病毒也有整合到宿主细胞基因组的可 能性。有人使用 $2 \mathrm{~A}$ 肽多蛋白表达载体, 即在一个病 毒载体中将 4 个诱导基因串联起来，使 4 种诱导因 子在单个启动子驱动下表达，从而减少了基因组中 病毒整合的位点数, 降低了插入突变的概率 ${ }^{[9]}$ 。在以 质粒为载体建立的iPS细胞形成的嵌合体小鼠中，没 有发现肿瘤, 但诱导iPS细胞所用的时间明显延长, 诱导效率明显下降。利用PiggyBac(PB)转座子携带 诱导因子产生iPS细胞, 也有成功的报道, 并在所获 得的iPS细胞中瞬时表达转座酶, 可将外源DNA片 段切除, 理论上可以获得没有基因组DNA改变的iPS 细胞, 但检测发现这种切除是不完全的 ${ }^{[34]}$ 。

小分子化合物和蛋白质都不是以核酸为基础的 载体, 没有导致插入突变的风险。在培养基中添加

表 2 诱导因子导入方法的比较

\begin{tabular}{|c|c|c|}
\hline 导入方式[文献] & 优点 & 缺点 \\
\hline 逆转录病毒 $[1]$ & 诱导效率高, 在干细胞中沉默 & 只适用于分裂细胞, 随机整合入基因组 \\
\hline 慢病毒 $[5,33]$ & 诱导效率高, 分裂和不分裂的细胞都能导入 & 随机整合入基因组 \\
\hline 质粒 ${ }^{[15]}$ & 无病毒成分, 基因整合频率低 & 诱导效率低, 需多轮转化, 随机整合入基因组 \\
\hline 转座子 $[34]$ & 适用多种类型体细胞, 能切除转基因 & 可能导致插入突变, 转基因移除不完全 \\
\hline 腺病毒 ${ }^{[35]}$ & 低频率基因组整合 & 诱导效率低, 易产生四倍体 \\
\hline 小分子化合物 ${ }^{[36]}$ & 控制诱导过程灵活 & 非特异性作用，遗传毒性 \\
\hline 蛋白质 37$]$ & 不以核酸为基础, 直接导入转录因子 & 某些蛋白纯化困难, 半衰期短 \\
\hline
\end{tabular}


小分子化合物, 如 5-氮杂胞苷(5-AZA)、BIX01294 (G9a组蛋白甲基化转移酶抑制剂) [38]、2-丙基戊酸 (即VPA, 组蛋白乙酰基转移酶抑制剂) ${ }^{[36]}$ 和钙通道 激动剂(BayK8644)等，则能通过抑制基因组的甲基 化, 或者影响特定的信号转导通路, 而显著提高iPS 细胞的诱导效率, 并能使诱导过程中产生的中间过 渡型细胞和不完全重编程的细胞转化为稳定的完全 重编程的多能干细胞。利用蛋白质分子诱导iPS细胞, 由于避免了遗传修饰, 因此安全性较高。但由于蛋 白质在细胞内半衰期短, 因此需要对体细胞进行多 次的蛋白质处理。此外, 蛋白质分子的诱导效率低、 诱导所需时间长 ${ }^{[11,39]}$, 制备大量高纯度的蛋白质因 子和把蛋白质因子有效地转运至细胞内也比较困 难。

\section{1 .3 诱导因子}

Oct3/4、Sox2、Klf4 和cMyc已被成功地用于将 多种小鼠和人体细胞诱导为iPS细胞 $11,6,13,15,30,40]$ 。 一般认为, Oct $3 / 4$ 和Sox2 是诱导多能性所需的核心 因子, 而Klf4 和 $c M y c$ 则能提高Oct3/4 和Sox2 的诱导 效率 ${ }^{[41]}$ 。但需要指出的是, 这些诱导因子与肿瘤的 发生关系密切。

$\mathrm{Kim}$ 等 ${ }^{[42]}$ 认为 $O c t 3 / 4$ 是由体细胞诱导产生iPS细 胞唯一不可缺少的因子, 但Oct $3 / 4$ 可以剂量依赖性 地决定ES细胞的致癌潜能 ${ }^{[43]}$ 。Oct3/4 的过量表达, 在大约 $25 \%$ 的人类实体肿瘤中均可检测到, 与肿瘤 的发生和不良预后密切相关 ${ }^{[40,44]}$ 。由于高达 $92 \%$ 的 人类实体瘤都具有染色体异常 ${ }^{[2]}$, 因此, 这些发现 提示Oct3/4 在诱导iPS细胞的过程中, 很可能也会诱 发iPS细胞产生遗传改变。

Sox2 通常作为协同因子参与Oct3/4 介导的基因 表达调控, 是多能性细胞的一种标记分子。Sox2 是 把不表达内源性Sox2 的体细胞诱导为iPS细胞时不 可或缺的因子 ${ }^{[42]}$ 。Sox2 过量表达, 在 $20 \%$ 的实体肿 瘤中均可检测到, 且与癌症的不良预后相关, 提示 其在肿瘤发生中起作用 ${ }^{[44,45]}$ 。但由于Sox2 对于iPS 细胞的诱导至关重要, 因此, 在诱导过程中, 必须 注意这个蛋白的表达水平, 以确保iPS细胞的遗传安 全性。

Klf4 在将体细胞诱导为iPS细胞过程中, 能上调 多能性基因Nanog, 并对染色质结构进行修饰, 从而
促进Oct3/4 和Sox2 与其靶序列的结合 $[40,46,47]$ 。但 Klf4 也具有与致癌信号转导蛋白相结合刺激细胞增 殖的作用, 提示Klf4 也是一个致癌因子 ${ }^{[42,47]}$ 。Klf4 在多种肿瘤中均高表达, 且与晚期癌症相关 $[44,48]$ 。 因此, 需要严格评估Klf4 对于诱导过程的必要性, 谨慎使用。

$c M y c$ 能诱导组蛋白乙酰化, 促进Oct3/4 和Sox2 结合到其靶基因上 ${ }^{[40,49]}$ 。 $c M y c$ 能提高端粒酶的活性, 使诱导过程中不会由于随细胞分裂端粒缩短而发生 细胞老化, 从而提高把体细胞诱导成为iPS细胞的效 率, 这对于端粒较短的体细胞来说尤显重要 ${ }^{[50]}$ 。 $c M y c$ 是著名的原癌基因, 能促进细胞增殖和转化, 且在多种肿瘤中, 都检测到 $c M y c$ 的高表达 ${ }^{[44]}$ 。值得 注意的是, Okita等 ${ }^{[26]}$ 发现, 在用 $c M y c$ 诱导产生的iPS 细胞形成的嵌合体小鼠中, 由于所转入的 $c M y c$ 的重 新活化导致了 $25 \%$ 的嵌合体小鼠发生了致死性的畸 胎瘤。Nakagawa等 ${ }^{[27]}$ 也发现，先用Oct4、Sox2、Klf4 和 $c M y c$ 诱导小鼠胚胎成纤维细胞形成iPS细胞, 这 些iPS细胞形成的嵌合体小鼠中, 约 $50 \%$ 在出生后 1 年内发生了肿瘤, 并在这些肿瘤中检测到 $\mathrm{cMyc}$ 的再 活化; 而在用Oct4、Sox2 和Klf4 诱导产生的iPS细胞 所形成的嵌合体小鼠中则没有观察到肿瘤的发生。 研究表明, 无 $c M y c$ 的诱导因子组合诱导iPS细胞时, 需要较长的诱导时间, 且诱导效率低, 但特异性较 强 ${ }^{[27]}$, 说明 $c M y c$ 的作用可能是促进细胞的增殖而不 是细胞的重编程 ${ }^{[51]}$ 。基于以上研究结果, 将 $c M y c$ 从 诱导因子组合中去除是可行的, 也能显著降低由iPS 细胞形成的嵌合体小鼠的肿瘤发生率。另外, Nakagawa等 ${ }^{[52]}$ 研发现, $L M y c$ 能提高iPS细胞的诱导效 率, 且 $L M y c$ 诱导形成的iPS细胞产生的嵌合体小鼠 较无 $c M y c$ 参与诱导形成的iPS细胞产生的嵌合体小 鼠, 虽死亡率略微升高, 但肿瘤发生率没有差异, 因此建议可以用 $L M y c$ 代替 $c M y c$ 进行诱导。

总之, 迄今用于诱导iPS细胞的所有因子几乎都 与肿瘤发生有关, 因此, 使用这些因子时需要特别 小心。第一, 首先确定哪个因子是体细胞诱导为iPS 细胞所必需的, 然后再用其对该体细胞进行诱导。 这样可避免非必需因子在起始细胞中的过表达、以 及在iPS细胞中的再活化致癌的风险 $[1,13,26]$ 。第二, 激 活内源性的 $c M y c 、 K l f 4$ 和Sox2 的表达可减少把神经 干细胞诱导为iPS细胞所需的诱导因子的数 量 ${ }^{[42]}$ 。 
从遗传安全性考虑, 用于iPS细胞诱导的最佳方案是 减少利用诱导因子, 并可人为调节这些诱导因子的 活性。

\section{$3.2 \mathrm{iPS}$ 细胞的培养}

大量研究表明, 在传代培养过程中, 染色体正 常的人ES细胞会自发转变为非整倍体细胞 ${ }^{[53]}$ 。在 传代培养的hiPS细胞中，会重复出现一些特定的染 色体异常, 如 12 号染色体短臂(12p)或整条 12 号染 色体的增加，以及 20 号染色体的亚区的扩增 [21]。我 们的研究结果也表明, 多个 miPS细胞系在体外培养 过程中, 重复出现 14 号染色体的三体和/或罗伯逊 易位的染色体异常 ${ }^{[25]}$ 。这提示iPS细胞的传代培养条 件还需进一步优化, 在临床治疗中, 应尽可能使用 早代的iPS细胞。

\section{4 展 望}

大量的研究结果表明, 迄今产生的多种 iPS 细 胞在遗传上并不安全, 无法满足临床应用的要求。 这一方面提示我们，必须利用更先进的实验技术来 检测 iPS 细胞中是否存在遗传学和表观遗传学异常, 另一方面，也要求研发新的诱导技术、避免使用可 能导致 iPS 细胞遗传改变的因子, 从而获得遗传上 安全的 iPS 细胞, 为 iPS 细胞的临床应用奠定基础。

\section{参考文献(References):}

[1] Takahashi K, Yamanaka S. Induction of pluripotent stem cells from mouse embryonic and adult fibroblast cultures by defined factors. Cell, 2006, 126(4): 663-676. DOI

[2] Weaver BA, Cleveland DW. Does aneuploidy cause cancer? Curr Opin Cell Biol, 2006, 18(6): 658-667. DOI

[3] Mitelman F, Johansson B, Mertens F. Mitelman database of chromosome aberrations in cancer. 2007. http://cgapncinihgov/ Chromosomes/Mitelman. DOI

[4] Yu JY, Vodyanik MA, Smuga-Otto K, AntosiewiczBourget J, Frane JL, Tian SL, Nie J, Jonsdottir GA, Ruotti V, Stewart R, Slukvin II, Thomson JA. Induced pluripotent stem cell lines derived from human somatic cells. Science, 2007, 318(5858): 1917-1920. DOI

[5] Blelloch R, Venere M, Yen J, Ramalho-Santos M. Generation of induced pluripotent stem cells in the absence of drug selection. Cell Stem Cell, 2007, 1(3): 245-247. DOI

[6] Park IH, Zhao R, West JA, Yabuuchi A, Huo HG, Ince TA, Lerou PH, Lensch MW, Daley GQ. Reprogramming of human somatic cells to pluripotency with defined factors. Nature, 2008, 451(7175): 141-146. DOI

[7] Li CL, Yu HY, Ma Y, Shi GL, Jiang J, Gu JJ, Yang Y, Jin SB, Wei Z, Jiang H, Li JS, Jin Y. Germline-competent mouse-induced pluripotent stem cell lines generated on human fibroblasts without exogenous leukemia inhibitory factor. PLoS One, 2009, 4(8): e6724. DOI

[8] Yu JY, Hu KJ, Smuga-Otto K, Tian SL, Stewart R, Slukvin II, Thomson JA. Human induced pluripotent stem cells free of vector and transgene sequences. Science, 2009, 324(5928): 797-801. DOI

[9] Carey BW, Markoulaki S, Hanna J, Saha K, Gao Q, Mitalipova M, Jaenisch R. Reprogramming of murine and human somatic cells using a single polycistronic vector. Proc Natl Acad Sci USA, 2009, 106(1): 157-162. DOI

[10] Li WL, Wei W, Zhu SY, Zhu JL, Shi Y, Lin TX, Hao EG, Hayek A, Deng HK, Ding S. Generation of rat and human induced pluripotent stem cells by combining genetic reprogramming and chemical inhibitors. Cell Stem Cell, 2009, 4(1): 16-19. DOI

[11] Kim D, Kim CH, Moon JI, Chung YG, Chang MY, Han BS, Ko S, Yang E, Cha KY, Lanza R, Kim KS. Generation of human induced pluripotent stem cells by direct delivery of reprogramming proteins. Cell Stem Cell, 2009, 4(6): 472-476. DOI

[12] Cho HJ, Lee CS, Kwon YW, Paek JS, Lee SH, Hur J, Lee EJ, Roh TY, Chu IS, Leem SH, Kim Y, Kang HJ, Park YB, Kim HS. Induction of pluripotent stem cells from adult somatic cells by protein-based reprogramming without genetic manipulation. Blood, 2010, 116(3): 386-395. DOI

[13] Wernig M, Meissner A, Foreman R, Brambrink T, Ku M, Hochedlinger $\mathrm{K}$, Bernstein $\mathrm{BE}$, Jaenisch $\mathrm{R}$. In vitro reprogramming of fibroblasts into a pluripotent ES-celllike state. Nature, 2007, 448(7151): 318-324. DOI

[14] Mali P, Ye ZH, Hommond HH, Yu XB, Lin J, Chen GB, Zou JZ, Cheng LZ. Improved efficiency and pace of generating induced pluripotent stem cells from human adult and fetal fibroblasts. Stem Cells, 2008, 26(8): 19982005. DOI

[15] Stadtfeld M, Nagaya M, Utikal J, Weir G, Hochedlinger K. Induced pluripotent stem cells generated without viral integration. Science, 2008, 322(5903): 945-949. DOI

[16] Mayshar Y, Ben-David U, Lavon N, Biancotti JC, Yakir B, Clark AT, Plath K, Lowry WE, Benvenisty N. Identification and classification of chromosomal aberrations in human induced pluripotent stem cells. Cell Stem Cell, 2010, 7(4): 521-531. DOI

[17] Minina IuM, Zhdanova NS, Shilov AG, Tolkunova EN, 
Liskovykh MA, Tomilin AN. Chromosomal instability of in vitro cultured mouse embryonic stem cells and induced pluripotent stem cells. Tsitologiia, 2010, 52(5): 420-425. $\underline{\text { DOI }}$

[18] Sommer CA, Sommer AG, Longmire TA, Christodoulou C, Thomas DD, Gostissa M, Alt FW, Murphy GJ, Kotton DN, Mostoslavsky G. Excision of reprogramming transgenes improves the differentiation potential of iPS cells generated with a single excisable vector. Stem Cells, 2010, 28(1): 64-74. DOI

[19] Gore A, Li Z, Fung HL, Young JE, Agarwal S, AntosiewiczBourget J, Canto I, Giorgetti A, Israel MA, Kiskinis E, Lee JH, Loh YH, Manos PD, Montserrat N, Panopoulos AD, Ruiz S, Wilbert ML, Yu JY, Kirkness EF, Izpisua Belmonte JC, Rossi DJ, Thomson JA, Eggan K, Daley GQ, Goldstein LSB, Zhang K. Somatic coding mutations in human induced pluripotent stem cells. Nature, 2011, 471 (7336): 63-67. DOI

[20] Hussein SM, Batada NN, Vuoristo S, Ching RW, Autio R, Närvä E, $\mathrm{Ng} \mathrm{S}$, Sourour M, Hämäläinen R, Olsson C, Lundin K, Mikkola M, Trokovic R, Peitz M, Brüstle O, Bazett-Jones DP, Alitalo K, Lahesmaa R, Nagy A, Otonkoski T. Copy number variation and selection during reprogramming to pluripotency. Nature, 2011, 471(7336): 58-62. DOI

[21] Laurent LC, Ulitsky I, Slavin I, Tran H, Schork A, Morey R, Lynch C, Harness JV, Lee S, Barrero MJ, Ku S, Martynova M, Semechkin R, Galat V, Gottesfeld J, Izpisua Belmonte JC, Murry C, Keirstead HS, Park HS, Schmidt U, Laslett AL, Muller FJ, Nievergelt CM, Shamir $\mathrm{R}$, Loring JF. Dynamic changes in the copy number of pluripotency and cell proliferation genes in human ESCs and iPSCs during reprogramming and time in culture. Cell Stem Cell, 2011, 8(1): 106-118. DOI

[22] Lister R, Pelizzola M, Kida YS, Hawkins RD, Nery JR, Hon G, Antosiewicz-Bourget J, O'Malley R, Castanon R, Klugman S, Downes M, Yu R, Stewart R, Ren B, Thomson JA, Evans RM, Ecker JR. Hotspots of aberrant epigenomic reprogramming in human induced pluripotent stem cells. Nature, 2011, 471(7336): 68-73. DOI

[23] Pasi CE, Dereli-Öz A, Negrini S, Friedli M, Fragola G, Lombardo A, Van Houwe G, Naldini L, Casola S, Testa G, Trono D, Pelicci PG, Halazonetis TD. Genomic instability in induced stem cells. Cell Death Differ, 2011, 18(5): 745-753. $\underline{\mathrm{DOI}}$

[24] Peterson SE, Westra JW, Rehen SK, Young H, Bushman DM, Paczkowski CM, Yung YC, Lynch CL, Tran HT, Nickey KS, Wang YC, Laurent LC, Loring JF, Carpenter
MK, Chun J. Normal human pluripotent stem cell lines exhibit pervasive mosaic aneuploidy. PLoS One, 2011, 6(8): e23018. DOI

[25] Chen Q, Shi XY, Rudolph C, Yu Y, Zhang D, Zhao XY, Mai S, Wang G, Schlegelberger B, Shi QH. Recurrent trisomy and Robertsonian translocation of chromosome 14 in murine iPS cell lines. Chromosome Res, 2011, 19(7): 857-868. DOI

[26] Okita K, Ichisaka T, Yamanaka S. Generation of germlinecompetent induced pluripotent stem cells. Nature, 2007, 448(7151): 313-317. DOI

[27] Nakagawa M, Koyanagi M, Tanabe K, Takahashi K, Ichisaka T, Aoi T, Okita K, Mochiduki Y, Takizawa N, Yamanaka S. Generation of induced pluripotent stem cells without Myc from mouse and human fibroblasts. Nat Biotechnol, 2008, 26(1): 101-106. DOI

[28] Nagata S, Toyoda M, Yamaguchi S, Hirano K, Makino H, Nishino K, Miyagawa Y, Okita H, Kiyokawa N, Nakagawa M, Yamanaka S, Akutsu H, Umezawa A, Tada T. Efficient reprogramming of human and mouse primary extra-embryonic cells to pluripotent stem cells. Genes Cells, 2009, 14(12): 1395-1404. DOI

[29] Maherali N, Hochedlinger K. Guidelines and techniques for the generation of induced pluripotent stem cells. Cell Stem Cell, 2008, 3(6): 595-605. DOI

[30] Aoi T, Yae K, Nakagawa M, Ichisaka T, Okita K, Takahashi K, Chiba T, Yamanaka S. Generation of pluripotent stem cells from adult mouse liver and stomach cells. Science, 2008, 321(5889): 699-702. DOI

[31] Shi QH, Chen JF, Adler ID, Zhang JX, Martin R, Pan SJ, Zhang XR, Shan XN. Increased nondisjunction of chromosome 21 with age in human peripheral lymphocytes. Mutat Res, 2000, 452(1): 27-36. DOI

[32] Fenech M, Bonassi S. The effect of age, gender, diet and lifestyle on DNA damage measured using micronucleus frequency in human peripheral blood lymphocytes. Mutagenesis, 2011, 26(1): 43-49. DOI

[33] Brambrink T, Foreman R, Welstead GG, Lengner CJ, Wernig M, Suh H, Jaenisch R. Sequential expression of pluripotency markers during direct reprogramming of mouse somatic cells. Cell Stem Cell, 2008, 2(2): 151-159. $\underline{\mathrm{DOI}}$

[34] Woltjen K, Michael IP, Mohseni P, Desai R, Mileikovsky M, Hämäläinen R, Cowling R, Wang W, Liu PT, Gertsenstein M, Kaji K, Sung HK, Nagy A. piggyBac transposition reprograms fibroblasts to induced pluripotent stem cells. Nature, 2009, 458(7239): 766-770. DOI

[35] Stadtfeld M, Maherali N, Breault DT, Hochedlinger K. 
Defining molecular cornerstones during fibroblast to iPS cell reprogramming in mouse. Cell Stem Cell, 2008, 2(3): 230-240. DOI

[36] Okita $K$, Nakagawa $M$, Hyenjong $H$, Ichisaka $T$, Yamanaka S. Generation of mouse induced pluripotent stem cells without viral vectors. Science, 2008, 322(5903): 949-953. DOI

[37] Gump JM, Dowdy SF. TAT transduction: the molecular mechanism and therapeutic prospects. Trends Mol Med, 2007, 13(10): 443-448. DOI

[38] Shi Y, Do JT, Desponts C, Hahm HS, Schöler HR, Ding S. A combined chemical and genetic approach for the generation of induced pluripotent stem cells. Cell Stem Cell, 2008, 2(6): 525-528. DOI

[39] Zhou HY, Wu SL, Joo JY, Zhu SY, Han DW, Lin TX, Trauger S, Bien G, Yao SS, Zhu Y, Siuzdak G, Schöler HR, Duan LX, Ding S. Generation of induced pluripotent stem cells using recombinant proteins. Cell Stem Cell, 2009, 4(5): 381-384. DOI

[40] Yamanaka S. Strategies and new developments in the generation of patient-specific pluripotent stem cells. Cell Stem Cell, 2007, 1(1): 39-49. DOI

[41] Boyer LA, Lee TI, Cole MF, Johnstone SE, Levine SS, Zucker JP, Guenther MG, Kumar RM, Murray HL, Jenner RG, Gifford DK, Melton DA, Jaenisch R, Young RA. Core transcriptional regulatory circuitry in human embryonic stem cells. Cell, 2005, 122(6): 947-956. DOI

[42] Kim JB, Sebastiano V, Wu GM, Araúzo-Bravo MJ, Sasse P, Gentile L, Ko K, Ruau D, Ehrich M, van den Boom D, Meyer J, Hübner K, Bernemann C, Ortmeier C, Zenke M, Fleischmann BK, Zaehres H, Schöler HR. Oct4-induced pluripotency in adult neural stem cells. Cell, 2009, 136(3): 411-419. DOI

[43] Gidekel S, Pizov G, Bergman Y, Pikarsky E. Oct-3/4 is a dose-dependent oncogenic fate determinant. Cancer Cell, 2003, 4(5): 361-370. DOI

[44] Schoenhals M, Kassambara A, De Vos J, Hose D, Moreaux J, Klein B. Embryonic stem cell markers expression in cancers. Biochem Biophys Res Commun, 2009, 383(2): 157-162. DOI

[45] Ben-Porath I, Thomson MW, Carey VJ, Ge RP, Bell GW, Regev A, Weinberg RA. An embryonic stem cell-like gene expression signature in poorly differentiated aggressive human tumors. Nat Genet, 2008, 40(5): 499-507. DOI

[46] Lin TX, Chao C, Saito S, Mazur SJ, Murphy ME, Appella E, Xu Y. p53 induces differentiation of mouse embryonic stem cells by suppressing Nanog expression. Nat Cell Biol, 2005, 7(2): 165-171. DOI

[47] Rowland BD, Bernards R, Peeper DS. The KLF4 tumour suppressor is a transcriptional repressor of p53 that acts as a context-dependent oncogene. Nat Cell Biol, 2005, 7(11): 1074-1082. DOI

[48] Foster KW, Liu ZL, Nail CD, Li XN, Fitzgerald TJ, Bailey SK, Frost AR, Louro ID, Townes TM, Paterson AJ, Kudlow JE, Lobo-Ruppert SM, Ruppert JM. Induction of KLF4 in basal keratinocytes blocks the proliferation-differentiation switch and initiates squamous epithelial dysplasia. Oncogene, 2005, 24(9): 1491-1500. DOI

[49] Fernandez PC, Frank SR, Wang LQ, Schroeder M, Liu SX, Greene J, Cocito A, Amati B. Genomic targets of the human c-Myc protein. Genes Dev, 2003, 17(9): 1115-1129. $\underline{\mathrm{DOI}}$

[50] Marion RM, Strati K, Li H, Tejera A, Schoeftner S, Ortega S, Serrano M, Blasco MA. Telomeres acquire embryonic stem cell characteristics in induced pluripotent stem cells. Cell Stem Cell, 2009, 4(2): 141-154. DOI

[51] Pera MF, Hasegawa K. Simpler and safer cell reprogramming. Nat Biotechnol, 2008, 26(1): 59-60. DOI

[52] Nakagawa $M$, Takizawa $N$, Narita $M$, Ichisaka $T$, Yamanaka S. Promotion of direct reprogramming by Transformation-deficient Myc. Proc Natl Acad Sci USA, 2010, 107(32): 14152-14157. DOI

[53] Baker DEC, Harrison NJ, Maltby E, Smith K, Moore HD, Shaw PJ, Heath PR, Holden H, Andrews PW. Adaptation to culture of human embryonic stem cells and oncogenesis in vivo. Nat Biotechnol, 2007, 25(2): 207-215.DOI 